\title{
Neurotoxic Lesions of the Medial Mediodorsal Nucleus of the Thalamus Disrupt Reinforcer Devaluation Effects in Rhesus Monkeys
}

\author{
Anna S. Mitchell, Philip G. F. Browning, and Mark G. Baxter \\ Department of Experimental Psychology, Oxford University, Oxford OX1 3UD, United Kingdom
}

\begin{abstract}
The mediodorsal thalamus is a major input to the prefrontal cortex and is thought to modulate cognitive functions of the prefrontal cortex. Damage to the medial, magnocellular part of the mediodorsal thalamus (MDmc) impairs cognitive functions dependent on prefrontal cortex, including memory. The contribution of MDmc to other aspects of cognition dependent on prefrontal cortex has not been determined. The ability of monkeys to adjust their choice behavior in response to changes in reinforcer value, a capacity impaired by lesions of orbital prefrontal cortex, can be tested in a reinforcer devaluation paradigm. In the present study, rhesus monkeys with bilateral neurotoxic MDmc lesions were tested in the devaluation procedure. Monkeys learned visual discrimination problems in which each rewarded object is reliably paired with one of two different food rewards and then were given choices between pairs of rewarded objects, one associated with each food. Selective satiation of one of the food rewards reduces choices of objects associated with that food in normal monkeys. Monkeys with bilateral neurotoxic lesions of MDmc learned concurrently presented visual discrimination problems as quickly as unoperated control monkeys but showed impaired reinforcer devaluation effects. This finding suggests that the neural circuitry for control of behavioral choice by changes in reinforcer value includes MDmc.
\end{abstract}

Key words: amygdala; choice behavior; decision-making; devaluation; medial thalamus; orbitofrontal cortex; prefrontal cortex; reward

\section{Introduction}

The prefrontal cortex (PFC) is involved in multiple aspects of memory, decision making, and cognitive control. Thalamic input to the PFC is thought to be involved in modulating its functions. Bilateral lesions of the medial, magnocellular part of the mediodorsal thalamus (MDmc) impair learning of object-in-place scenes in rhesus monkeys (Gaffan and Parker, 2000) (Mitchell et al., 2007), a task dependent on PFC (Browning et al., 2005). MDmc interacts with both the temporal lobe and ventromedial PFC in rapid learning of single visual discrimination problems in a discrimination learning set paradigm (Gaffan and Murray, 1990; Gaffan et al., 1993). These findings are congruent with the viewpoint that removal of input from MDmc to PFC disables the cognitive functions of PFC (Gaffan and Parker, 2000).

The PFC is also involved in decision making and cognitive control. One function dependent on the PFC, specifically orbital PFC, is the capacity to adjust choice behavior in response to a change in reinforcer value. This adaptive response selection has tremendous survival value because it allows organisms to make choices based on the current value of a reinforcer rather than its history. The neural circuitry for this ability in monkeys, tested in

Received April 27, 2007; revised Aug. 24, 2007; accepted Aug. 27, 2007.

This work was supported by the Medical Research Council (A.S.M., P.G.F.B.; programme grant to Prof. David Gaffan) and the Wellcome Trust (M.G.B.). We thank D. Kyriazis for technical support.

Correspondence should be addressed to Dr. Anna S. Mitchell, Department of Experimental Psychology, Oxford University, South Parks Road, Oxford, OX1 3UD, UK.

DOI:10.1523/JNEUROSCI.1914-07.2007

Copyright $\odot 2007$ Society for Neuroscience $\quad 0270-6474 / 07 / 2711289-07 \$ 15.00 / 0$ a reinforcer devaluation paradigm, includes the amygdala and lateral orbital PFC (Malkova et al., 1997; Baxter et al., 2000; Izquierdo et al., 2004; Machado and Bachevalier, 2007) but does not require intact entorhinal and perirhinal cortex (Thornton et al., 1998). The MDmc is interconnected with all of these brain regions. Basolateral amygdala and entorhinal and perirhinal cortex project to MDmc (Aggleton and Mishkin, 1984; Russchen et al., 1987; Saunders et al., 2005). The MDmc has prominent reciprocal connections with ventral and ventromedial orbitofrontal cortex (Goldman-Rakic and Porrino, 1985; Giguere and Goldman-Rakic, 1988; Ray and Price, 1993). The contribution of MDmc to reinforcer devaluation performance has not been determined in nonhuman primates. If $\mathrm{MDmc}$ is required for the normal functioning of PFC in response to any demands placed on it, bilateral MDmc lesions would be expected to impair reinforcer devaluation effects just as bilateral orbital prefrontal lesions do.

In the present study, we trained rhesus monkeys, with either bilateral neurotoxic lesions of MDmc or intact controls, in the reinforcer devaluation paradigm (Malkova et al., 1997). Monkeys learned 60 visual discrimination problems, presented concurrently once in each training session. Each rewarded object (the correct object in each pair) was consistently associated with one of two different food rewards (a half-peanut or sugar-coated chocolate candy). Once discrimination problems were learned, sessions of critical trials were given in which the 60 rewarded objects were randomly paired to provide 30 choice trials between an object paired with a half-peanut and an object paired with chocolate. Before some of these critical trial sessions, each mon- 
key was allowed to consume one of the two foods to satiety. Normal monkeys make fewer choices of the objects paired with the devalued food after this selective satiation procedure, but this effect is impaired by bilateral lesions of amygdala, orbital PFC, or surgical disconnection of these structures (Malkova et al., 1997; Baxter et al., 2000; Izquierdo et al., 2004; Machado and Bachevalier, 2007).

\section{Materials and Methods}

Subjects. These were nine rhesus monkeys (Macaca mulatta), seven male and two female. They lived in troops, separated by sex, in large indoor enclosures attached to standard caging. Water was available ad libitum in the home cage, and each monkey's daily food ration was given at the completion of the training session of each day. They were behaviorally sophisticated at the time of reinforcer devaluation training. All had been trained on object-in-place scene learning (Gaffan, 1994). Seven of the monkeys [MD1, MD2, MD3 (monkeys receiving lesions of the magnocellular mediodorsal thalamus), CON1, CON2, CON3, and CON4 (control monkeys)] had also been trained on strategy implementation (Gaffan et al., 2002) (Mitchell et al., 2007) as well as concurrent discrimination learning, reversal of concurrent discriminations, and learning and serial reversal of a single visual discrimination problem, all with clip-art stimuli similar to those used in the present study. All of these tasks used $190 \mathrm{mg}$ banana-flavored or sugar pellets (P. J. Noyes, Lancaster, NH) as rewards. Half-peanut and M\&Ms were only used as rewards for behavioral test performance in the context of reinforcer devaluation training and selective satiation, although all monkeys were familiar with these foods before they began this training. MDmc lesions were produced between 4 and 6.5 months before the beginning of training on the visual discrimination problems for reinforcer devaluation testing in monkeys MD1-MD5. CON1-CON3 did not undergo any neurosurgical procedures or anesthesia before or during the present study. CON4 received sterile saline injections into ventrolateral prefrontal cortex. This monkey, operated as part of a separate study of selective neurochemical lesions of ventrolateral prefrontal cortex, was not intended to control for surgical manipulations of the MDmc but rather provided an additional control case that had undergone anesthesia and neurosurgery but without sustaining any neuronal damage.

Apparatus. All behavioral testing took place in an automated apparatus, as described in other publications (Browning et al., 2005). Clip-art stimuli were presented on a touch-sensitive screen $(380 \times 280 \mathrm{~mm})$ located $\sim 150 \mathrm{~mm}$ from the front of the transport cage. The monkey could reach through horizontally oriented bars on the front of the transport cage to touch the screen and obtain rewards. M\&Ms and half-peanuts were delivered by automated feeders (Med Associates, St. Albans, VT) to a food cup located centrally below the touch screen. On the completion of each test session, the monkey was given its daily food by the experimenter and allowed to consume it while still in the transport cage. A clear plastic "lunchbox" $(\sim 200 \times 100 \times 100 \mathrm{~mm})$ that could be attached to the front of the transport cage was used for the specific satiation.

Behavioral testing. This task followed procedures described by Malkova et al. (1997) and Baxter et al. (2000) except that it took place in an automated apparatus instead of a manual one. A set of 60 pairs of clip-art objects, each pair constituting a problem, comprised the visual discrimination problems. One of the two clip-art objects was arbitrarily designated correct in each pair. The objects were presented against a gray background, one on the left side of the screen and one on the right, which was randomized across trials. Touching the correct object resulted in the incorrect object disappearing, delivery of a reward pellet, and then the correct object disappearing after $1 \mathrm{~s}$. Touching the incorrect object caused both objects to disappear immediately and no reward was delivered. The intertrial interval was $30 \mathrm{~s}$ after a choice was made, and a touch to the screen during the intertrial interval reset it. Each problem appeared once in each session.

Half of the rewarded objects resulted in the delivery of a half-peanut, and the other rewarded objects produced an M\&M chocolate candy. Training continued until a criterion of 270 or more correct responses over five consecutive sessions ( $90 \%$ or greater correct) was reached. At this point, a series of sessions of critical trials were presented in which the
60 rewarded objects were randomly assigned to create 30 pairs of critical trials, each offering a choice between a peanut-rewarded object and an M\&M-rewarded object. Some sessions of critical trials were preceded by a devaluation procedure in which the monkey was allowed to consume one of the two food rewards to satiation before beginning the critical trial session. For the devaluation, the monkey was moved into the transport cage and remained in the housing room. The plastic lunchbox was affixed to the front of the cage containing a known amount of food reinforcer (either M\&Ms or peanuts). The monkey was left undisturbed for $15 \mathrm{~min}$ to consume the food. If the food was completely eaten, the box was refilled. The monkey was then observed closely, and, once it had not taken any food for $5 \mathrm{~min}$, the box was removed from the cage. Once the monkey's cheek pouches were not visibly full of food, it was moved to the testing cubicle and the critical trial session begun. The sequence of critical trial sessions was baseline, peanut devaluation, baseline, M\&M devaluation, and was repeated once. Each critical trial session was separated by at least one standard training session, and monkeys had at least $2 \mathrm{~d}$ of rest after a critical trial session in which devaluation occurred. It is important to note that, during these critical trial sessions when one of the foods has been satiated, no new learning of object-reward associations can occur because each pair of objects followed by a food reward is only presented once during the critical trial session.

The critical measure of performance was a score composed of the difference in number of choices of objects paired with a particular food on baseline sessions and in sessions when that food was devalued. These scores were added together for each devalued food. This was calculated separately for each sequence of critical trial sessions, and the mean was taken as the overall score. For example, a monkey that chose $12 \mathrm{M} \& \mathrm{M}$ objects and 18 peanut objects in the baseline sessions (mean of the two baseline sessions) then chose five peanut objects when peanuts were devalued and seven M\&M objects when M\&Ms were devalued would have a difference score of $(18-5)+(12-7)=18$. If he chose $14 \mathrm{M} \& \mathrm{M}$ objects and 16 peanut objects in baseline sessions of the second set of critical trial sessions and then three peanut objects and seven M\&M objects when each was devalued, this would give a score of 20 for the second set of critical sessions and a difference score of 19 overall.

Monkeys ate an average $98 \mathrm{~g}$ of food in the selective satiation procedure and spent an average of $15 \mathrm{~min}$ in the devaluation sessions. Neither the amounts eaten nor the time spent differed between the groups $(t$ values $<1.46$; $p$ values $>0.05$ ).

Surgery. Neurosurgical procedures were performed in a dedicated operating theater under aseptic conditions. Monkeys were given steroids (MD1 and MD2, $1 \mathrm{mg} / \mathrm{kg}$ dexamethasone; MD3-MD5, methylprednisolone at $20 \mathrm{mg} / \mathrm{kg}$ ) the night before surgery (intramuscularly), and two (MD1 and MD2, dexamethasone at $1-2 \mathrm{mg} / \mathrm{kg}$ ) or three (MD3MD5, CON4, methylprednisolone at $20 \mathrm{mg} / \mathrm{kg}$ ) doses were given intravenously or intramuscularly on the day of surgery to protect against intraoperative edema and postoperative inflammation. Each monkey was sedated on the morning of surgery with both ketamine $(10 \mathrm{mg} / \mathrm{kg})$ and xylazine $(0.5 \mathrm{mg} / \mathrm{kg})$. Once sedated, the monkey was given atropine $(0.05 \mathrm{mg} / \mathrm{kg}$ ) to reduce secretions, antibiotic (amoxicillin at $8.75 \mathrm{mg} / \mathrm{kg}$ ) for prophylaxis of infection, opioid (buprenorphine at $0.01 \mathrm{mg} / \mathrm{kg}$, i.v., repeated twice at $4-6 \mathrm{~h}$ intervals on the day of surgery, i.v. or i.m.) and nonsteroidal anti-inflammatory (meloxicam at $0.2 \mathrm{mg} / \mathrm{kg}$, i.v.) agents for analgesia, and a histamine $\mathrm{H}_{2}$ receptor antagonist (ranitidine at $1 \mathrm{mg} / \mathrm{kg}$, i.v.) to protect against gastric ulceration as a side effect of the combination of steroid and nonsteroidal anti-inflammatory treatment. The head was shaved and an intravenous cannula put in place for intraoperative delivery of fluids (warmed sterile saline drip, $5 \mathrm{ml} \cdot \mathrm{h}^{-1} \cdot \mathrm{kg}^{-1}$ ). The monkey was moved into the operating theater, intubated, placed on isoflurane (MD1, MD2, MD4, MD5, and CON4, $1.2-2.7 \%$, to effect, in $100 \%$ oxygen) or sevoflurane (MD3, 2.25-4.0\%, to effect, in 100\% oxygen), and then mechanically ventilated. Adjustable heating blankets allowed maintenance of normal body temperature during surgery. Heart rate, oxygen saturation of hemoglobin, mean arterial blood pressure, end-tidal $\mathrm{CO}_{2}$, body temperature, and respiration rate were monitored continuously throughout surgery.

The monkey was placed in a stereotaxic head holder, and the head was cleaned with alternating antimicrobial scrub and alcohol and draped to 
allow a midline incision. The skin and underlying galea were opened in layers. The temporal muscles were retracted as necessary to expose the skull surface over the intended lesion site. A D-shaped bone flap was turned over the right hemisphere and the midline. The dura over the posterior part of the right hemisphere was cut and retracted to the midline. The right hemisphere was retracted with a brain spoon, and the splenium of the corpus callosum was cut in the midline with a glass aspirator. The tela choroidea (the membrane covering the third ventricle and the thalamus) was cauterized in the midline posterior and dorsal to the thalamus using a metal aspirator that was insulated to the tip. The posterior commissure, the third ventricle posterior to the thalamus, and the most posterior $5 \mathrm{~mm}$ of the midline thalamus were exposed. A stereotaxic manipulator holding a $10 \mu \mathrm{l}$ Hamilton syringe with a blunttipped 26-gauge needle with its hole $1 \mathrm{~mm}$ above the base was positioned above the posterior commissure at the midline using the third ventricle as a guide. Neurotoxic bilateral lesions to the magnocellular division of the mediodorsal thalamic nucleus were produced by $10 \times 1 \mu$ injections of a mixture of ibotenic acid $(10 \mathrm{mg} / \mathrm{ml}$; Biosearch Technologies, Novato, CA) and NMDA (10 mg/ml; Tocris Bioscience, Bristol, UK) dissolved in sterile 0.1 м PBS. The monkey brain atlas of Ilinsky and Kultas-Ilinsky (1987) was used to calculate the intended lesion site coordinates. The needle was positioned for the first pair of coordinates [anteroposterior (AP), $+5.2 \mathrm{~mm}$ anterior to the posterior commissure; mediolateral (ML), $\pm 1.2 \mathrm{~mm}$ lateral to the third ventricle; dorsoventral (DV), -4.0 $\mathrm{mm}$ (to compensate for the hole positioned $1 \mathrm{~mm}$ above the tip of the needle)] ventral to the surface of the thalamus directly above the intended lesion site. Each injection was made slowly over $4 \mathrm{~min}$, and the needle was left in place for $\sim 4$ min before being moved to the next site. The needle was then repositioned for the second pair of coordinates: AP, $+4.2 \mathrm{~mm}$; ML, $\pm 1.5 \mathrm{~mm}$; DV, $-5.0 \mathrm{~mm}$. The third, fourth, and fifth pairs of coordinates were as follows: $\mathrm{AP},+4.2 \mathrm{~mm}, \mathrm{ML}, \pm 1.5 \mathrm{~mm}$, and DV, $-3.0 \mathrm{~mm}$; AP, $+3.4 \mathrm{~mm}, \mathrm{ML}, \pm 1.7 \mathrm{~mm}$, and DV, $-4.0 \mathrm{~mm}$; and $\mathrm{AP},+3.4$ $\mathrm{mm}, \mathrm{ML}, \pm 1.7 \mathrm{~mm}$, and DV,$-3.0 \mathrm{~mm}$, respectively. In each case, the DV coordinate was relative to the surface of the thalamus at the injection site. CON4 received a total of 34 (17 per hemisphere) handheld $1 \mu$ sterile PBS injections into ventrolateral prefrontal cortex. The boundaries for these injections extended from the ventral lip of the principal sulcus to the fundus of the lateral orbital sulcus. The anterior and posterior limits were lines joining the tips of the principal and lateral orbital sulci.

When the lesions were complete, the dura was repositioned over the brain but not sewn, the bone flap was replaced and held with loose sutures, and the skin and galea were closed in layers. Mechanical ventilation was discontinued intermittently until the monkey was breathing spontaneously; end-tidal $\mathrm{CO}_{2}$ and oxygen saturation of hemoglobin were closely monitored during this process to prevent hypoxia or hypercapnia. The monkey was removed from the head holder, and anesthesia was discontinued. The monkey was extubated when a swallowing reflex was observed, returned to the home cage, and monitored continuously until normal posture was regained. Nonsteroidal anti-inflammatory analgesic (meloxicam, $0.2 \mathrm{mg} / \mathrm{kg}$, oral) and antibiotic $(8.75 \mathrm{mg} / \mathrm{kg}$, oral) treatment continued after surgery in consultation with veterinary staff, typically for $5 \mathrm{~d}$. Each monkey was returned to its social group as soon as practical after surgery, usually the next day.

Histology. After completion of all behavioral testing, each monkey was sedated with ketamine $(10 \mathrm{mg} / \mathrm{kg})$, deeply anesthetized with intravenous barbiturate, and transcardially perfused with $0.9 \%$ saline followed by $10 \%$ formalin. The brains were cryoprotected in formalin-sucrose and then sectioned coronally on a freezing microtome at $50 \mu \mathrm{m}$ thickness. A 1-in-10 series of sections was collected throughout the thalamus that was expanded to a 1 -in- 5 series through the area of the lesion; these were mounted on gelatin-coated glass microscope slides and stained with cresyl violet.

Figures 1 and 2 show schematic diagrams depicting the medial thalamus as well as photomicrographs of cresyl violet-stained coronal sections corresponding as closely as possible to these schematic diagrams for the five MDmc-lesioned monkeys (MD1, MD2, MD3, MD4, and MD5). All five monkeys in the MDmc group had extensive bilateral lesions in the magnocellular division of the mediodorsal thalamic nucleus as intended. The largest lesions were in monkeys MD1, MD3, and MD4, with slight sparing in the most rostral and caudal extents of the MDmc. The MDmc damage in MD2 and MD5 was slightly smaller than the other three lesions, with more extensive atrophy in these lesions within the caudal aspects of the MDmc. In the more rostral parts of MDmc, the dorsal area was almost completely spared in these two monkeys. MD2 also sustained some slight damage to the right posterior cingulate and right retrosplenial cortex. MD1, MD3, and MD5 also sustained unilateral damage to the anterior thalamic nuclei that extended into the anterior portion of the laterodorsal thalamus in MD1 and MD5. Other thalamic damage outside the MDmc was similar in all five lesions within the rhomboid and central medial nuclei. The paraventricular nucleus of the epithalamus was extensively damaged throughout the entire anteroposterior extent of the lesions. All five monkeys also had sagittal section of the splenium of the corpus callosum and hippocampal commissure dorsal to the posterior thalamus.

\section{Results}

Monkeys with bilateral neurotoxic lesions of MDmc learned the discrimination problems at a similar rate as controls: mean \pm SEM sessions to criterion, control, $19.3 \pm 3.8$; MD, $14.0 \pm 0.86$ (including the criterion run). During the baseline preference testing, there were no significant differences in the object and food pairings selected ( $t$ values $<1.41$; $p$ values $>0.05$ ) (Fig. $3 A$ ).

In contrast to their intact learning of the discrimination problems that formed the critical trials for devaluation testing, MDlesioned monkeys showed attenuated devaluation effects relative to controls. Although the difference scores, on average, were higher for the second devaluation test than the first (mean of 18.88 vs 15.55 ), this difference was not statistically significant in the entire sample of monkeys $\left(t_{(8)}=1.62 ; p=0.13\right)$, nor was it significant in either control or MD monkeys considered separately ( $t$ values $<1.70 ; p$ values $>0.19$ ). Thus, our main measure of performance was the mean difference score across the two devaluation tests, although we also considered both separately. This measure is plotted in Figure $3 B$, and the data from devaluation tests are presented in Table 1. MD-lesioned monkeys were impaired in devaluation as measured by the overall difference score $\left(t_{(7)}=2.34 ; p=0.03\right.$, one-tailed). This impairment was more prominent on the second devaluation test (comparison of performance on the second test only, $t_{(7)}=3.43 ; p=0.01$, one-tailed) and was not significant on the first test considered alone $\left(t_{(7)}=1.31 ; p=0.12\right.$, one-tailed). Presumably, this reflects the fact that the range of performance is larger on the second devaluation test, because all monkeys tended to show higher devaluation scores when the test was repeated, congruent with previous investigations with this task (Baxter et al., 2000; Izquierdo and Murray, 2004a).

We note that both groups showed difference scores higher than chance performance (an expected value of 0 ): controls, $t_{(3)}$ $=8.49, p=0.003 ; \mathrm{MD}, t_{(4)}=5.21, p=0.006$. Thus, although monkeys with MDmc lesions are impaired relative to controls in devaluation performance, they still show performance that is superior to what would be expected based on chance.

\section{Discussion}

Bilateral neurotoxic lesions to the MDmc impaired the ability to adjust choice behavior in response to changes in the reinforcer value. Previous work has shown similar effects after bilateral lesions of orbital PFC (Izquierdo et al., 2004), amygdala (Malkova et al., 1997), or crossed lesions of the amygdala and orbital PFC (Baxter et al., 2000). We predicted deficits in reward devaluation after MDmc lesions because of interconnections between the amygdala, orbital PFC, and the MDmc (Aggleton and Mishkin, 1984; Goldman-Rakic and Porrino, 1985; Russchen et al., 1987; Giguere and Goldman-Rakic, 1988). Thus, the current findings provide important evidence regarding a link between the MDmc, the amygdala, and the orbital PFC interacting within a neural circuit involved in mediating re- 


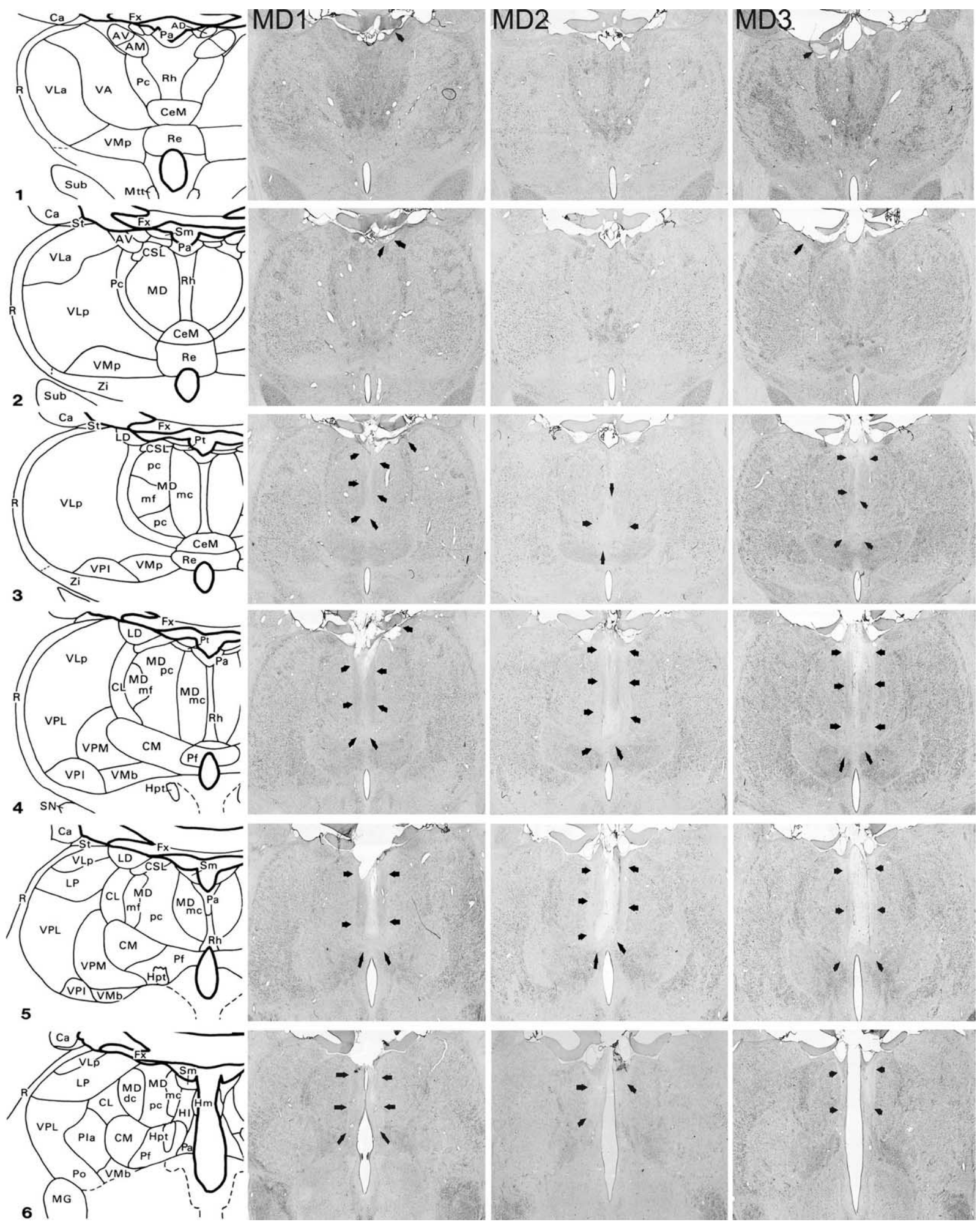

Figure 1. MDmc lesions. Schematic diagrams of six sections, $1 \mathrm{~mm}$ apart, through the posterior thalamus of a monkey taken from Gaffan and Murray (1990). The next three columns show photomicrographs of the MDmc lesions for MD1-MD3 corresponding as closely as possible to the each of the schematic diagrams. (Photomicrographs of MD4 and MD5 are shown in Figure 2.) Arrowheads indicate the site of each lesion. AD, Anterodorsal nucleus; $\mathrm{AM}$, anteromedial nucleus; $\mathrm{AV}$, anteroventral nucleus; $\mathrm{Ca}$, caudate nucleus; $\mathrm{CeM}$, central medial nucleus; $\mathrm{CL}$, central lateral nucleus; $\mathrm{CM}$, center median nucleus; $\mathrm{CSL}$, central superior lateral nucleus; $\mathrm{FC}$, frontal cortex; $\mathrm{FX}$, fornix; HI, lateral habenular nucleus; $\mathrm{Hm}$, medial habenular nucleus; Hpt, habenulopeduncular tract; IT, inferotemporal cortex; LD, lateral dorsal nucleus; Li, limitans nucleus; LP, lateral posterior nucleus; MD, mediodorsal nucleus; MDdc, mediodorsal nucleus, densocellular portion; MDmc, mediodorsal nucleus, magnocellular portion; MDmf, mediodorsal nucleus, multiformis portion; MDpc, mediodorsal nucleus, parvocellular portion; $M G$, medial geniculate complex; $M m$, mamillary complex; $M t t$, mamillothalamic tract; $P a$, paraventricular nucleus; $P c$, paracentral nucleus; Pf, parafascicular nucleus; PFC, prefrontal cortex; Pla, anterior pulvinar nucleus; Pli, inferior pulvinar nucleus; Pl, lateral pulvinar nucleus; Po, posterior nucleus; Pa, parataenial nucleus; R, reticular nucleus; Re, reuniens nucleus; Rh, rhomboid nucleus; SG, suprageniculate nucleus; SN, substantia nigra; St, stria terminalis; Sub, subthalamic nucleus; VA, ventral anterior nucleus; VLa, ventral lateral nucleus, anterior portion; VLp, ventral lateral nucleus, posterior portion; VMb, ventral medial nucleus, basal portion; VMp, ventral medial nucleus, principal portion; VPi, ventral posterior inferior nucleus; VPI, ventral posterior lateral nucleus; VPm, ventral posterior medial nucleus; Zi, zona incerta. 

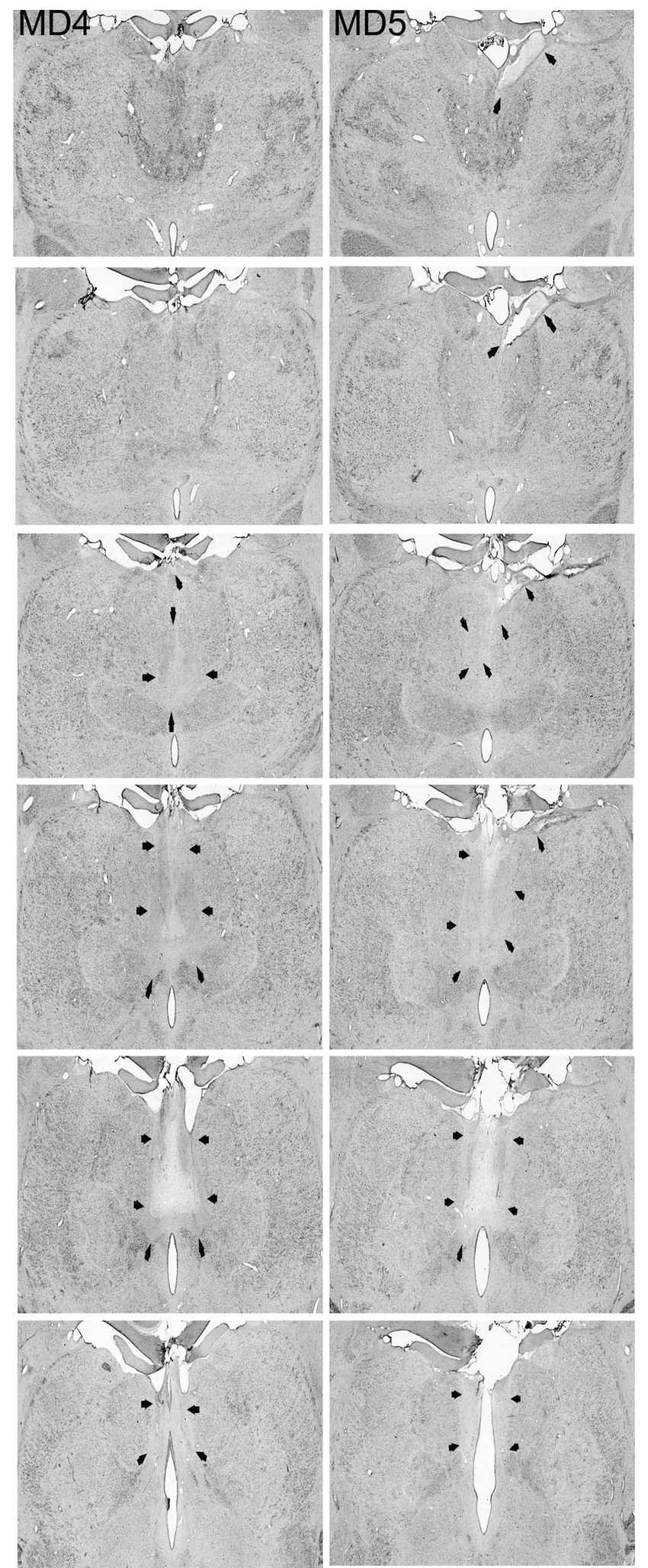

Figure 2. MDmc lesions continued. The two columns show photomicrographs of the MDmc lesions for MD4 and MD5 corresponding as closely as possible to the each of the schematic diagrams shown in Figure 1. Arrowheads indicate the site of each lesion.

sponse selection and goal-directed behavior. This relationship is reinforced by a crossed lesion study of unilateral MDmc lesions in one hemisphere combined with unilateral orbital PFC and amygdala lesions in the other hemisphere, which also impairs reinforcer deval-
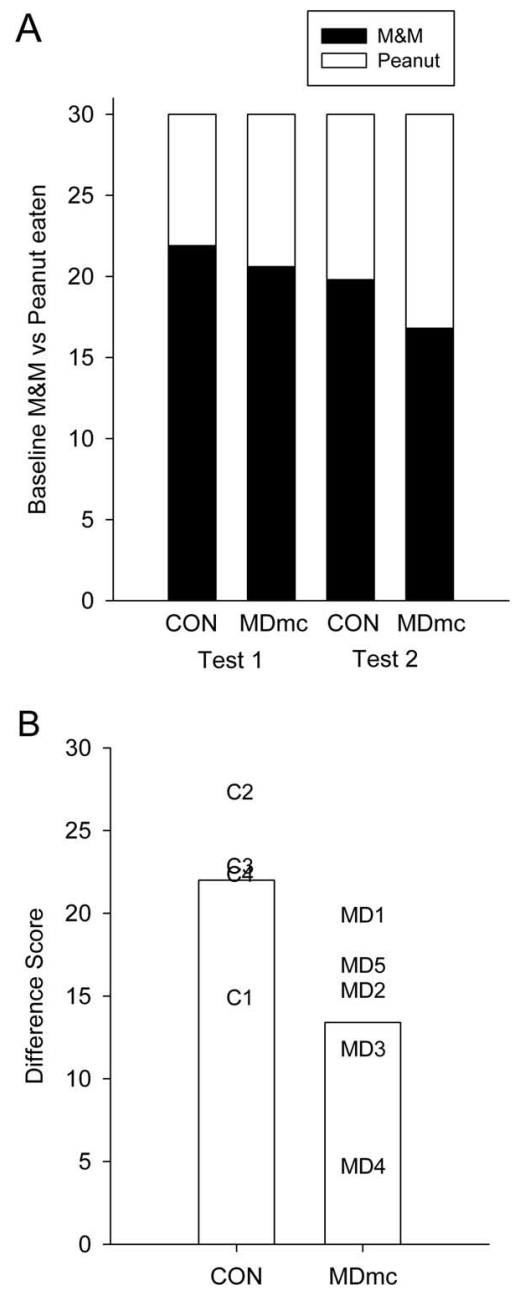

Figure 3. Devaluation. $A$, Mean number of objects selected based on either M\&M or peanut rewards during baseline preference test sessions of test 1 and test 2 for neurotoxic magnocellular mediodorsal thalamus lesion (MDmc) and unoperated control (CON) monkeys. $\boldsymbol{B}$, Mean difference scores across the two devaluation tests for MDmc and CON monkeys. Data presented are from individual monkeys.

uation (Izquierdo and Murray, 2004b). The dorsal midline thalamic nuclei, including the paraventricular and parataenial nuclei, may have also contributed to the current deficits in reward devaluation because these two nuclei are also interconnected with orbital prefrontal cortex (Hsu and Price, 2007) and they were unavoidably damaged when making the MDmc neurotoxic lesions.

The current results cannot be explained by deficiencies in visual perception or learning abilities because there were no group differences in initial acquisition of the 60 object pairs. It also appears that the MDmc monkeys and controls demonstrated reliable food preferences because performance during baseline testing indicated that most monkeys consistently chose food 1 (M\&Ms) objects over food 2 (peanut) objects (Table 1). Thus, the operated group were aware of the values of the reinforcers and their relationships with the specific objects. Furthermore, all of the monkeys had participated as subjects in other cognitive tests before the devaluation paradigm and demonstrated dissociable cognitive performance between impaired scene learning and an almost normal level of proficiency on a preoperatively acquired strategy implementation task in monkeys MD1-MD3 (Mitchell, Baxter, and Gaffan, unpublished observations) or impaired new scene learning versus normal retention of 300 preoperatively ac- 
Table 1. Devaluation performance for the two tests

\begin{tabular}{|c|c|c|c|c|c|c|c|c|c|}
\hline \multirow[b]{2}{*}{ Case } & \multicolumn{4}{|l|}{ Test 1} & \multicolumn{4}{|l|}{ Test 2} & \multirow[b]{2}{*}{ Mean } \\
\hline & Baseline, M:P & Satiate M, M:P & Satiate $P, M: P$ & Difference score & Baseline, M:P & Satiate $M, M: P$ & Satiate $P, M: P$ & Difference score & \\
\hline CON1 & $30: 0$ & $21: 9$ & $30: 0$ & 9 & $30: 0$ & $9: 21$ & $30: 0$ & 21 & 15 \\
\hline CON2 & $18.5: 11.5$ & $2: 28$ & $30: 0$ & 28 & $11: 19$ & $0: 30$ & $27: 3$ & 27 & 27.5 \\
\hline CON3 & $18: 12$ & $1: 29$ & 19:11 & 18 & $18: 12$ & $2: 28$ & $30: 0$ & 28 & 23 \\
\hline CON4 & $21: 9$ & $7: 23$ & $29: 1$ & 22 & $20: 10$ & $6: 24$ & $29: 1$ & 23 & 22.5 \\
\hline Mean & 21.9:8.1 & $7.8: 22.2$ & $27: 3$ & 19.25 & $19.8: 10.2$ & $4.3: 25.7$ & $29: 1$ & 24.75 & 22 \\
\hline MD1 & $21: 9$ & $8: 22$ & $27: 3$ & 19 & $15.5: 14.5$ & $5: 25$ & $25: 5$ & 20 & 19.5 \\
\hline MD2 & $19.5: 10.5$ & $16: 14$ & $27: 3$ & 11 & $13.5: 16.5$ & $6: 24$ & $25: 5$ & 19 & 15 \\
\hline MD3 & $16: 14$ & $10: 20$ & $21: 9$ & 11 & $17.5: 12.5$ & $11 ; 19$ & $23: 7$ & 12 & 11.5 \\
\hline MD4 & $29.5: 0.5$ & $27: 3$ & $29: 1$ & 2 & $25.5: 4.5$ & $20: 10$ & $27: 3$ & 7 & 4.5 \\
\hline MD5 & $17: 13$ & $6: 24$ & $26: 4$ & 20 & $12: 18$ & $6: 24$ & 19:11 & 13 & 16.5 \\
\hline Mean & 20.6:9.4 & $13.4: 16.6$ & $26: 4$ & 12.6 & $16.8: 13.2$ & 9.6:20.4 & $23.8: 6.2$ & 14.2 & 13.4 \\
\hline
\end{tabular}

The number of M\&M (M) and peanut (P) objects chosen by each monkey during the baseline sessions (mean of 2 baseline sessions for each test) and for sessions preceded by devaluation of either peanuts or M\&Ms.

quired scenes in monkeys MD4 and MD5 (Mitchell et al., 2007). Thus, the MDmc monkeys did not lack motivation to work for the food rewards. Instead, the results of the current study suggest that MDmc-lesioned monkeys, like orbital PFC- and amygdalalesioned monkeys, were unable to adapt their behavior when responding to a choice between two visual objects, which had both been previously rewarding immediately after changes to the value of one of the reinforcers.

It is perhaps surprising that neurotoxic MDmc lesions were without effect on learning the concurrent visual discrimination (object-reward association) problems that formed the pairs of objects used for reinforcer devaluation testing. Aspiration lesions of MDmc impair object-reward association learning (Gaffan and Parker, 2000) as do bilateral lesions of the prefrontal cortex (Parker and Gaffan, 1998). It should be noted that the monkeys with MDmc lesions in the present study were very sophisticated behaviorally by the time these problems were presented (as were the controls), which may mitigate the deficit produced by MDmc ablation in object-reward association learning. Moreover, deficits in object-reward association learning may be less reliable or severe after MDmc lesions relative to impairments in other learning tasks such as scene learning (Mitchell et al., 2007). Bilateral lesions of the ventrolateral or orbital PFC, which reliably impair scene learning, do not impair concurrent object-reward association learning (our unpublished observations).

The range of deficits (Table 1) after bilateral MDmc lesions in the current study were not as severe as those reported after bilateral lesions of orbital PFC (Izquierdo et al., 2004) or amygdala (Malkova et al., 1997) or disconnection of these structures (Baxter et al., 2000). The comparison of magnitude of impairment with these studies is not straightforward, however, because our controls also had higher devaluation scores than previously reported controls (Izquierdo et al., 2004). The impairment resulting from MDmc lesions in the present study does appear to be a milder impairment than that of monkeys with bilateral orbital PFC lesions tested in precisely the same paradigm (our unpublished observations). Furthermore, the current testing procedure is not directly comparable with these previously reported studies because they used a manual apparatus, whereas the current study used an automated testing design. Nevertheless, the milder deficits resulting from MDmc lesions suggest that the MDmc role in the neural circuitry associated with response control may be more regulatory rather than essential. The neuroanatomical connections of the circuit support this proposal, whereby the amygdala and orbital PFC continue to communicate via a direct route (Porrino et al., 1981; Amaral and Price, 1984; Carmichael and Price, 1996).

The components that account for cognitive deficits after MDmc damage have not yet been identified. Our laboratory has proposed recently that the interactions between $\mathrm{PFC}$ and MDmc are critical in rapid new learning (Mitchell et al., 2007). However, the critical trials during the devaluation procedure do not involve new learning because all of the rewarded objects are only presented once during this critical trial session, so there is no opportunity to form new associations between an object and the now-devalued reinforcer. It could be argued that the impairment is related to a deficit in flexible responding or cognitive flexibility more generally. Rats with MD lesions also have reversal learning deficits (Chudasama et al., 2001). However, the effects of lesions on reversal learning and reinforcer devaluation are not identical. Orbital PFC lesions impair both (Izquierdo et al., 2004), but neurotoxic amygdala lesions impair reinforcer devaluation (Malkova et al., 1997) and not reversal learning (Izquierdo and Murray, 2007), whereas rhinal cortex lesions impair reversal learning (Murray et al., 1998) and not reinforcer devaluation (Thornton et al., 1998). Thus, it is not apparent that the involvement of MDmc in reinforcer devaluation can be ascribed with confidence to an impairment in new learning or a generalized impairment in cognitive flexibility.

Interestingly, devaluation deficits in an instrumental conditioning paradigm have been shown in rats with mediodorsal thalamus lesions (Corbit et al., 2003) but not with orbitofrontal cortex lesions (Ostlund and Balleine, 2007). Instead, orbitofrontal cortex lesions only caused impairments in outcome encoding during pavlovian conditioning (Ostlund and Balleine, 2007). Basolateral amygdala lesions impair reinforcer devaluation in both instrumental and pavlovian conditioning paradigms (Hatfield et al., 1996; Balleine et al., 2003; Pickens et al., 2003; Corbit and Balleine, 2005). These results suggest that the brain structures interconnected in this neural circuitry are not simply processing information in the same way, although lesions of amygdala, orbitofrontal cortex, and MDmc all impair devaluation performance in this procedure in monkeys. Rather the subcortical structures, namely the mediodorsal thalamus and the amygdala, are involved with integration of information about pavlovian and instrumental outcomes, whereas orbitofrontal cortex is more selectively engaged depending on task demands. The design of our task, at present, does not allow us to distinguish between the contribution of pavlovian and instrumental processes to performance.

A preliminary report that crossed unilateral lesions of $\mathrm{MDmc}$ in one hemisphere and amygdala and OFC in the other impair reinforcer devaluation effects (Izquierdo and Murray, 2004b) suggests that the involvement of MDmc in this task is via its interaction with these structures. Perhaps the hypothesized involvement of MDmc in PFC-dependent new learning (Mitchell et al., 2007) extends to the modulation of amygdala-orbital PFC 
interactions in the course of adjusting response selection during the reinforcer devaluation procedure, when choice behavior must be modified in response to the change in the value of the food reinforcer. This would also account for the apparently milder effect of MDmc lesions on reinforcer devaluation than that of bilateral ablation of orbital PFC or amygdala or their disconnection. Neurophysiological experiments examining neural correlates of reinforcer devaluation in orbital PFC and amygdala in the presence and absence of modulatory input from MDmc would go a long way toward elucidating this issue.

Finally, a role for the MDmc in this neural circuitry associated with response selection suggests that, just like humans with orbital PFC and amygdala damage, patients with damage in the mediodorsal thalamus may be impaired in using information about the likely consequences of their actions to guide their behavior. To our knowledge, relatively little research has investigated these aspects of medial thalamic brain damage. Recent studies have reported that Korsakoff's syndrome patients suffer impairments in affective judgments (Brand et al., 2003) and also make very poor choices and judgments in a laboratory-based gambling task (Brand et al., 2005). Nevertheless, in both reports, the authors propose that the deficits are mainly the result of frontal lobe pathology. The current findings now suggest that neuronal loss in the mediodorsal thalamic nucleus of Korsakoff's syndrome patients (Harding et al., 2000; Kopelman, 2002) may also contribute to the deficits in these affective judgments and irrational responding in gambling-based tasks directly.

\section{References}

Aggleton JP, Mishkin M (1984) Projections of the amygdala to the thalamus in the cynomolgus monkey. J Comp Neurol 222:56-68.

Amaral DG, Price JL (1984) Amygdalo-cortical projections in the monkey (Macaca fascicularis). J Comp Neurol 230:465-496.

Balleine BW, Killcross AS, Dickinson A (2003) The effect of lesions of the basolateral amygdala on instrumental conditioning. J Neurosci 23:666-675.

Baxter MG, Parker A, Lindner CC, Izquierdo AD, Murray EA (2000) Control of response selection by reinforcer value requires interaction of amygdala and orbital prefrontal cortex. J Neurosci 20:4311-4319.

Brand M, Fujiwara E, Kalbe E, Steingass HP, Kessler J, Markowitsch HJ (2003) Cognitive estimation and affective judgments in alcoholic Korsakoff patients. J Clin Exp Neuropsychol 25:324-334.

Brand M, Fujiwara E, Borsutzky S, Kalbe E, Kessler J, Markowitsch HJ (2005) Decision-making deficits of korsakoff patients in a new gambling task with explicit rules: associations with executive functions. Neuropsychology 19:267-277.

Browning PG, Easton A, Buckley MJ, Gaffan D (2005) The role of prefrontal cortex in object-in-place learning in monkeys. Eur J Neurosci 22:3281-3291.

Carmichael ST, Price JL (1996) Connectional networks within the orbital and medial prefrontal cortex of macaque monkeys. J Comp Neurol 371:179-207.

Chudasama Y, Bussey TJ, Muir JL (2001) Effects of selective thalamic and prelimbic cortex lesions on two types of visual discrimination and reversal learning. Eur J Neurosci 14:1009-1020.

Corbit LH, Balleine BW (2005) Double dissociation of basolateral and central amygdala lesions on the general and outcome-specific forms of pavlovian-instrumental transfer. J Neurosci 25:962-970.

Corbit LH, Muir JL, Balleine BW (2003) Lesions of mediodorsal thalamus and anterior thalamic nuclei produce dissociable effects on instrumental conditioning in rats. Eur J Neurosci 18:1286-1294.

Gaffan D (1994) Scene-specific memory for objects: a model of episodic memory impairment in monkeys with fornix transection. J Cogn Neurosci 6:305-320.

Gaffan D, Murray EA (1990) Amygdalar interaction with the mediodorsal nucleus of the thalamus and the ventromedial prefrontal cortex in stimulus reward associative learning in the monkey. J Neurosci 10:3479-3493.

Gaffan D, Parker A (2000) Mediodorsal thalamic function in scene memory in rhesus monkeys. Brain 123:816-827.

Gaffan D, Murray EA, Fabrethorpe M (1993) Interaction of the amygdala with the frontal-lobe in reward memory. Eur J Neurosci 5:968-975.
Gaffan D, Easton A, Parker A (2002) Interaction of inferior temporal cortex with frontal cortex and basal forebrain: double dissociation in strategy implementation and associative learning. J Neurosci 22:7288-7296.

Giguere M, Goldman-Rakic PS (1988) Mediodorsal nucleus: areal, laminar, and tangential distribution of afferents and efferents in the frontal lobe of rhesus monkeys. J Comp Neurol 277:195-213.

Goldman-Rakic PS, Porrino LJ (1985) The primate mediodorsal (MD) nucleus and its projection to the frontal lobe. J Comp Neurol 242:535-560.

Harding A, Halliday G, Caine D, Kril J (2000) Degeneration of anterior thalamic nuclei differentiates alcoholics with amnesia. Brain 123:141-154.

Hatfield T, Han JS, Conley M, Gallagher M, Holland P (1996) Neurotoxic lesions of basolateral, but not central, amygdala interfere with pavlovian second-order conditioning and reinforcer devaluation effects. J Neurosci 16:5256-5265.

Hsu DT, Price JL (2007) Midline and intralaminar thalamic connections with the orbital and medial prefrontal networks in macaque monkeys. J Comp Neurol 504:89-111.

Ilinsky IA, Kultas-Ilinsky K (1987) Sagittal cytoarchitectonic maps of the Macaca mulatta thalamus with a revised nomenclature of the motorrelated nuclei validated by observations on their connectivity. J Comp Neurol 262:331-364.

Izquierdo A, Murray EA (2004a) Combined unilateral lesions of the amygdala and orbital prefrontal cortex impair affective processing in rhesus monkeys. J Neurophysiol 91:2023-2039.

Izquierdo A, Murray EA (2004b) Mediodorsal nucleus of the thalamus contributes to reinforcer devaluation effects in rhesus monkeys. Soc Neurosci Abstr 30:84.9.

Izquierdo A, Murray EA (2007) Selective bilateral amygdala lesions in rhesus monkeys fail to disrupt object reversal learning. J Neurosci 27:1054-1062.

Izquierdo A, Suda RK, Murray EA (2004) Bilateral orbital prefrontal cortex lesions in rhesus monkeys disrupt choices guided by both reward value and reward contingency. J Neurosci 24:7540-7548.

Kopelman MD (2002) Disorders of memory. Brain 125:2152-2190.

Machado CJ, Bachevalier J (2007) The effects of selective amygdala, orbital frontal cortex or hippocampal formation lesions on reward assessment in nonhuman primates. Eur J Neurosci 25:2885-2904.

Malkova L, Gaffan D, Murray EA (1997) Excitotoxic lesions of the amygdala fail to produce impairment in visual learning for auditory secondary reinforcement but interfere with reinforcer devaluation effects in rhesus monkeys. J Neurosci 17:6011-6020.

Mitchell AS, Baxter MG, Gaffan D (2007) Dissociable performance on scene learning and strategy implementation after lesions to magnocellular mediodorsal thalamic nucleus. J Neurosci, in press.

Murray EA, Baxter MG, Gaffan D (1998) Monkeys with rhinal cortex damage or neurotoxic hippocampal lesions are impaired on spatial scene learning and object reversals. Behav Neurosci 112:1291-1303.

Ostlund SB, Balleine BW (2007) Orbitofrontal cortex mediates outcome encoding in pavlovian but not instrumental conditioning. J Neurosci 27:4819-4825.

Parker A, Gaffan D (1998) Memory after frontal/temporal disconnection in monkeys: conditional and non-conditional tasks, unilateral and bilateral frontal lesions. Neuropsychologia 36:259-271.

Pickens CL, Saddoris MP, Setlow B, Gallagher M, Holland PC, Schoenbaum G (2003) Different roles for orbitofrontal cortex and basolateral amygdala in a reinforcer devaluation task. J Neurosci 23:11078-11084.

Porrino LJ, Crane AM, Goldman-Rakic PS (1981) Direct and indirect pathways from the amygdala to the frontal lobe in rhesus monkeys. J Comp Neurol 198:121-136.

Ray JP, Price JL (1993) The organization of projections from the mediodorsal nucleus of the thalamus to orbital and medial prefrontal cortex in macaque monkeys. J Comp Neurol 337:1-31.

Russchen FT, Amaral DG, Price JL (1987) The afferent input to the magnocellular division of the mediodorsal thalamic nucleus in the monkey, Macaca fascicularis. J Comp Neurol 256:175-210.

Saunders RC, Mishkin M, Aggleton JP (2005) Projections from the entorhinal cortex, perirhinal cortex, presubiculum, and parasubiculum to the medial thalamus in macaque monkeys: identifying different pathways using disconnection techniques. Exp Brain Res 167:1-16.

Thornton JA, Malkova L, Murray EA (1998) Rhinal cortex ablations fail to disrupt reinforcer devaluation effects in rhesus monkeys (Macaca mulatta). Behav Neurosci 112:1020-1025. 